\title{
Dilation of the Giant Vortex State in a Mesoscopic Superconducting Loop.
}

\author{
S. Pedersen†, G.R. Kofod, J.C. Hollingbery, C.B. Sørensen, and P.E. Lindelof \\ The Niels Bohr Institute, University of Copenhagen, Universitetsparken 5, DK-2100 Copenhagen, \\ Denmark
}

November 1, 2018

\begin{abstract}
We have experimentally investigated the magnetisation of a mesoscopic aluminum loop at temperatures well below the superconducting transition temperature $T_{c}$. The flux quantisation of the superconducting loop was investigated with a $\mu$-Hall magnetometer in magnetic field intensities between \pm 100 Gauss. The magnetic field intensity periodicity observed in the magnetization measurements is expected to take integer values of the superconducting flux quanta $\Phi_{0}=h / 2 e$. A closer inspection of the periodicity, however, reveal a sub flux quantum shift. This fine structure we interpret as a consequence of a so called giant vortex state nucleating towards either the inner or the outer side of the loop. These findings are in agreement with recent theoretical reports.
\end{abstract}

PACS numbers: 74.60.Ec,74.25.Dw,73.23.-b,74.20.De,74.76.-w

Ever since the original observation and explanation of flux quantization 1 , the superconducting flux quanta $\Phi_{0}=h / 2 e$ has played a fundamental role in solid state physics. The concept of flux quantisation has been crucial for the interpretation of a wide range of classical condensed matter experiments, concerning e.g. weakly connected rings oscillations $\mathrm{G}^{\mathrm{G}}$. 
However, all these investigations were primarily performed at temperatures close to the critical temperature $T_{c}$ and at magnetic field intensities well below $H_{c 2}$. Recently it has become possible with $\mu$-Hall magnetometers to perform high resolution magnetisation experiments on small superconducting aluminium discs in the full magnetic field intensity range of superconductivity and at temperatures well below $T_{c}^{9}$ 目. These investigations have revealed information from deep within the superconducting phase, a regime which previously hasn't been accessible. Not unexpectedly these reports have attracted considerable interest also from a theoretical point of view $12-17,24-26$.

It is well known that for type-II $(\kappa=\lambda / \xi>1 / \sqrt{2})$ bulk superconductors a triangular Abrikosov vortex lattice is created in the magnetic field intensity range $H_{c 1}<H<H_{c 2}$ where $\kappa$ is the Ginzburg-Landau parameter, and $H_{c 1}$ and $H_{c 2}$ are the first and second critical fields. Since the effective Ginzburg-Landau parameter is significantly increased in thin films when the width of the film becomes comparable to the superconducting coherence length $\xi_{o}$, the appearance of an Abrikosov lattice is expected even in thin films consisting of type-I superconducting materials. When the spatial dimensions of the sample are decreased even further, and several length scales of the system become comparable with $\xi_{o}$, the competition between the Abrikosov vortex configuration and symmetry of the sample boundary becomes important. Hence for such mesoscopic systems the bulk critical fields $H_{c 1}$ and $H_{c 2}$ no longer are the only controlling parameters of the vortex configurations.

When considering sufficiently small superconducting rings the confinement effects from the boundaries are dominating and impose a circular symmetry on the superconducting order parameter. Hence the order parameter is expected to be given by $\psi(r)=F(r) e^{i L \theta}$ where $L$ is the angular momentum or vorticity of the vortex. When the superconductor is described by such a circular symmetric order parameter it is said to be in a giant vortex stat 2426 . In a recent theoretical work the properties of giant vortex states and multi-vortex states in mesoscopic superconducting discs and rings were treated extensively 24.25 . It was found that the giant vortex state indeed is energetic favorable in narrow rings due to the strong influence of the ring surface. Furthermore, the superconducting state can consist of a combination 
of the paramagnetic- and the diamagnetic Meissner state. In other words the direction of the supercurrents closest to the outer edge are opposite to the currents running closest to the inner edge. This means that at a certain effective radius between the outer and inner edge the supercurrent density goes to zero. Since this effective zero-current radius is the one that determines the area in which the flux is quantized, it becomes possible to measure this effective radius by studying the magnetization of superconducting mesoscopic loops. It was furthermore pointed out that when increasing the magnetic field intensity from zero field this effective radius would move towards the outer edge as a signature of the giant vortex state.

The measurement described in this paper was performed on a micron sized superconducting aluminium loop placed on top of a $\mu$-Hall magnetometer. The $\mu$-Hall magnetometer was etched out of a $\mathrm{GaAs} / \mathrm{Ga}_{0.7} \mathrm{Al}_{0.3} \mathrm{As}$ heterostructure. The mobility and electron density of the bare two-dimensional electron gas was $\mu=42 \mathrm{~T}^{-1}$ and $n=1.9 \times 10^{15} \mathrm{~m}^{-2}$. A symmetrical $4 \mu \mathrm{m} \times 4 \mu \mathrm{m}$ Hall geometry was defined by standard e-beam lithography on top of the heterostructure. In a later processing step a lift-off mask was defined on top of the $\mu$-Hall probe by e-beam lithography. After deposition of a $t=90 \mathrm{~nm}$ thick layer of aluminium and lift-off the sample looked as presented in Fig.1. The mean radius of the aluminium loop is $R=2.16 \mu \mathrm{m}$ and the average wire width $w$ is $316 \pm 40 \mathrm{~nm}$. The superconducting coherence length was estimated to be approximately $\xi_{o}=180 \mathrm{~nm}$ corresponding to a bulk critical field of $H_{c 2}=\Phi_{0} / 2 \pi \xi_{o}^{2} \approx 100$ Gauss.

By using the expression

$n \Phi_{0}=n \frac{h}{2 e}=\Delta\left(\mu_{0} H\right) \pi R^{2}$

where $A=\pi R^{2}$ is the area of the loop given by its mean radius $R$, it is found that a single flux jumps $(n=1)$ corresponds to a magnetic field periodicity given by $\Delta\left(\mu_{0} H\right)=1.412$ Gauss for the ring shown in Fig.11.

The samples was cooled in a ${ }^{3} \mathrm{He}$ cryostat equipped with a superconducting soleniode driven 
by a DC current supply. The magnetic field intensity was changed in steps of 57.7 mGauss. Measurements discussed here were performed in the temperature range between $T=0.3 \mathrm{~K}$ and the transition temperature of the superconducting loop $T_{c} \approx 1.2 \mathrm{~K}$.

The relation between the Hall voltage $V_{H}$ and the magnetic field intensity $H$ perpendicular to the $\mu$-Hall magnetometer is given by the classical Hall effect

$V_{H}=-\frac{I}{n e} \mu_{0}(H+\alpha M)$

were $I$ is the DC current through the $\mu$-Hall magnetometer and $\alpha$ is a dimensionless number of the order of unity, which corresponds to the ratio between the sensitive area of the $\mu$-Hall probe and the area of the object which is the source of the magnetisation $M 12$. 19 . For our superconducting rings we find that $\alpha$ typically was in the range between $0.3 \ldots 0.4$.

By using standard AC lock-in techniques, where the driving current $I$ was modulated, the Hall voltage $V_{H}$ was measured as a function of magnetic field intensity $\mu_{0} H$.

Similar results as the ones presented here were observed in several samples with identical dimensions in a number of cooldowns.

Also a circular loops with a width of $w=630 \mathrm{~nm}$, but with the same mean radius as the loops described above, were investigated.

In Fig.2. is displayed the measured local magnetsation $\mu_{0} M$ detected by the $\mu$-Hall probe as a function of magnetic field intensity $\mu_{0} H$. The measurement was performed at $T=0.36 \mathrm{~K}$ on the device presented in Fig.1. The curve displays a series of distinct jumps corresponding to the abrupt changes in magnetisation of the superconducting loop. The difference in magnetic field intensity between two successive flux jumps is approximately given by $\Delta\left(\mu_{0} H\right)=1.4$ Gauss or $\Delta\left(\mu_{0} H\right)=2.8$ Gauss which corresponds to either single or double flux jumps $(n=1$ or $n=2)$.

Large flux jumps $(n>1)$ or flux avalanches, occur whenever the system is trapped in a metastable state. It was generally observed that these flux avalanches become more pronounced with decreasing temperature, at low magnetic field intensities and for wide loops. Furthermore the flux avalanches were sensitive to the cooling procedure. 
The energy barrier causing the metastability of the eigenstates of the loop, are due to either the Beam-Livingston surface barrier or the volume barrier, or even an interplay of both $13,20.21$.

In Fig.3. the magnetic field intensity difference between successive jumps $\Delta\left(\mu_{0} H\right)$, in units of the 1.412Gauss (corresponding to a single superconducting flux quantum), have been plotted as a function of magnetic field intensity. It is seen that the magnetic field intensity difference between the observed jumps is, to a high accuracy, given by integer values of 1.412Gauss. At absolute magnetic field intensities lower than 40Gauss double flux jumps dominate, whereas at higher absolute magnetic field intensities only single flux jumps are observed. The figure shows both an up-sweep and a down-sweep as indicated by the arrows. Similar results obtained from the device with width $w=630 \mathrm{~nm}$ are presented in Fig.5. For these thicker loops it is seen that the flux avalanches are much more pronounced; avalanches corresponding to eleven single flux jumps were observed around zero magnetic field intensity. For these loops a gradual transition from huge flux avalanches $(n=11)$ to single flux jumps occur as the magnetic field intensity is increased - similar to the sharp transition between double and single flux jumps observed for the thinner loops.

In the graphs presented in Fig.3. it is seen that a small systematic variation of the value of the flux jumps occur when the magnetic field intensity is changed. This fine structure appears as a memory effect, in the sense that as the magnetic field intensity is increased (decreased) the size of the flux jumps decreases (increases). Thus these deviations are dependent, not only on the size of the magnetic field intensity, but also on which direction the magnetic field intensity was sweept during measurements. The data presented in Fig.3. has been replotted on Fig.⿴囗十 in the following way: We use Eq.(1) to calculate the effective radius $R$ of the superconducting loop and plot this radius as a function of magnetic field intensity. The dotted horizontal lines in Fig. 4 . represents the mean inner $R_{i}$ and outer radius $R_{o}$ determined from the SEM picture. It is seen that as the magnetic field intensity is changed from negative to positive values, the effective radius, as defined from the flux quantization condition of the loop, changes from inner to outer radius and vice versa. 
For a superconducting loop at low magnetic field intensities, it is expected that the appropriate effective radius is given by the geometrical mean value of the outer and inner radius $R=\sqrt{R_{i} R_{o} 222425}$. This is indeed in good agreement with the observed behavior around zero magnetic field intensity.

In the regime of high magnetic field intensities the concept of surface superconductivity becomes important and the giant vortex state will nucleate on the edges of the loop 24. In this regime two degenerate current carrying situations are possible 23 - hence the giant vortex state can either circulate the loop clockwise or anti-clockwise.

Since the orientation of the current in the loop is determined by the sweep direction (Lenz' law), a decreasing (increasing) magnetic field intensity will give rise to a anti-clockwise (clockwise) circulation. Hence as the magnetic field intensity is sweept from e.g. a large positive value to a large negative value the effective radius of the loop will change from inner to outer radius and vice versa giving rise to the observed memory effect.

The width of the giant vortex state is approximately given by the magnetic length $l_{H}=$ $\sqrt{\hbar / e H}[$. Hence any variation of the effective radius should take place over a magntic field range given by the condition that the width of the loop and the magnetic length are comparable; $w=l_{H}$. Such an estimate gives a characteristic magnetic field intensity of 34Gauss in good agreement with the presented data on Fig. 4 .

A similar effective radius analysis of the data presented on Fig. 5 . becomes rather dubious due to the combination of large flux avalanches and the larger width $w$.

At even larger magnetic field intensities $\left(\left|\mu_{o} H\right| \approx 60\right.$ Gauss $)$ the effective radius undergoes a transition from outer $R_{o}$ (or inner radius $R_{i}$ ) to the mean radius $R$. We speculate that this could be due a 2D-1D transition due to an increase in the superconducting coherence length $\xi_{o}$ with magnetic field intensity 26 .

The characteristic dimensionless parameter used to distinguish between a discs and loops is given by the ratio $x=R_{i} / R_{o}$ between outer and inner radius 24 26. In our case the thin loops have $x=0.86$, and for the thick loop to $x=0.75$.

In the recent works by two theoretical groups 2426 it is found that at large $x$ values (corre- 
sponding to a loop consisting of a one-dimensional wire) no or little variation of the effective radius should be observed. Whereas at small $x$ values (corresponding to a disc) a fast decrease of the effective radius occur as the magnetic field intensity increases. In the intermediate regime $x=0.5$, a rather smooth transition between average and outer radius should take place when the magnetic field intensity increases.

In the presented measurement for the thinner loop $(x=0.86)$, we indeed observe that the effective radius varies smoothly between inner and outer radius. This behavior looks similar to the one predicted for loops with $x=0.5$, however not similar to the one predicted expected for $x=0.75$. We do not find this discrepancy severe for the following reasons: The calculations by Bruyndoncx et al.26 were done using the linearised first Ginzburg-Landau equation, hence these results are only valid close to the phase transition, viz. $R_{o} / \xi_{o}<1$. In the work by Peeters et al.24 25 the full set of non-linear Ginzburg-Landau equations were solved self-consistently, in the two cases where $R_{o} / \xi_{o}=4$ and 2. Neither of these conditions were fulfilled in our experiments, where we estimate $R_{o} / \xi_{o} \approx 12$. It is furthermore seen by studying the results of Peeters et al. that calculations with larger values of $R_{o} / \xi_{0}$ probably would give rise to a better agreement.

For the thick loops $(x=0.75)$ we observed large flux avalanches at low magnetic field intensities. The large flux avalanches disguise any variation of the effective radius. Furthermore occurrence of flux avalanches in superconducting loops have not been dealt with quantitatively in the theoretical literature as far as the authors know. Hence comparisons with theory are not possible at the present time.

In summary, we present high resolution magnetisation measurements performed on superconducting aluminum loops. The resolution of the $\mu$-Hall magnetometer allowed us to resolve sub flux quantum effects and hence directly observe the dilation of a giant vortex state.

This work was financially supported by Velux Fonden, Ib Henriksen Foundation, Novo Nordisk Foundation, The Danish Research Council (grant 9502937, 9601677 and 9800243) and the Danish Technical Research Council (grant 9701490). The authors acknowledge Lars Melwyn Jensen, J. Berger, F. Peeters and V.V. Moshchalkov for discussions. 
$\nmid$ Present address: Department of Microelectronics and Nanoscience, Chalmers University of Technology, SE 41296 Göteborg, Sweden. 


\section{REFERENCES}

1. B.S. Deaver, Jr., and W. M. Fairbank, Phys. Rev. Lett. 7, 43 (1961).

2. R. Doll and M. Näbauer, Phys. Rev. Lett. 7, 51 (1961).

3. A.H. Silver and J.E. Zimmerman. Phys. Rev. 157, 317 (1967).

4. L.D. Jackel, W.W. Webb, J.E. Lukens, and S.S. Pei, Phys. Rev. B. 9, 115 (1974).

5. L.D. Jackel, R.A. Buhrmann, and W.W. Webb, Phys. Rev. B. 10, 2782 (1974).

6. W.A. Little and R.D. Parks. Phys. Rev. Lett. 9, 9 (1962).

7. R.D. Parks and W.A. Little. Phys. Rev. 133, A97 (1964).

8. R.P. Groff and R.D. Parks. Phys. Rev. 176, 567 (1968).

9. A.K. Geim, S.V. Dubonos, J.G.S. Lok, I.V. Grigorieva, J.C. Maan, L.T. Hansen, and P.E. Lindelof. Appl. Phys. Lett. 71, 2379 (1997).

10. A.K. Geim, I.V. Grigorieva, S.V. Dubonos, J.G.S. Lok, J.C. Maan, A.E. Filippov, and F.M. Peeters. Nature 390259 (1997).

11. A.K. Geim, S.V. Dubonos, J.G.S. Lok, M. Henini, and J.C. Maan. Nature 396144 (1998).

12. P. Singha Deo, V.A. Schweigert, F.M. Peeters, and A.K. Geim. Phys. Rev. Lett. 79 4653 (1997).

13. P. Singha Deo, V.A. Schweigert and F.M. Peeters, Phys. Rev. B. 596039 (1999).

14. V.A. Schweigert, F.M. Peeters. Phys. Rev. Lett. 812783 (1998).

15. V.A. Schweigert, and F.M. Peeters. Phys. Rev. B. 5713817 (1998).

16. J.J. Palacios. Phys. Rev. B. 58 R5948 (1998).

17. R. Benoist and W. Zwerger, Z. Phys. B 103 377-381 (1997) 
18. I.S. Ibrahim, V.A. Schweigert, and F.M. Peeters. Phys. Rev. B. 5715416 (1998).

19. F.M. Peeters and X.Q. Li. Appl. Phys. Lett. 72572 (1998)

20. C.P. Bean and J.D. Livingston, Phys. Rev. Lett. 12, 14 (1964)

21. X.Zhang and J.C. Price. Phys. Rev. B. 553128 (1997).

22. R.M. Arutunian and G.F. Zharkov. Journal of Low Temp. Phys. 52409 (1983).

23. See e.g. M.Tinkham, Introduction to superconductivity 2d ed. (McGraw-Hill 1996), p. 141-143.

24. F.M. Peeters, V.A. Schweigert, B.J. Baelus, and P.S. Deo. Physica C 332 255-262 (2000).

25. B.J. Baelus, F.M. Peeters, and V.A. Schweigert. Phys. Rev. B. 619734 (2000).

26. V. Bruyndoncx, L. Van Look, M. Verschuere, and V.V. Moshchalkov. Phys. Rev. B. 60 10468 (1999). 


\section{FIGURES}

Fig. 1. Scanning electron microscope image of a $\mu$-Hall probe, the cross section of the etched $\mu$-Hall probe is $4 \times 4 \mu \mathrm{m}^{2}$. The mean radius of the superconducting aluminium loop deposited on top of the $\mu$-Hall magnetometer is $2.16 \mu \mathrm{m}$, and the difference between the outer and inner radius is $314 \mathrm{~nm}$.

Fig. 2. Measured magnetisation $\mu_{0} M$ detected by the $\mu$-Hall probe as a function of magnetic field intensity $\mu_{0} H$ of the device presented on Fig.(何. The curve displays distinct jumps corresponding to the abrupt changes in magnetisation of the superconducting loop when the system changes state. The measurements were performed at $T=0.36 \mathrm{~K}$.

Fig. 3. The magnetic field intensity difference $\Delta\left(\mu_{0} H\right)$ between two successive jumps in magnetisation. Given in units of 1.412 Gauss corresponding to a single flux quantum $\Phi_{0}=h / 2 e$. The plotted jumps are given as a function of magnetic field intensity. The measurement was performed at $T=0.36 \mathrm{~K}$. The positive (negative) flux values corresponds to the case where $\mu_{0} H$ was decreased (increased) during the measurements. Arrows indicate sweep direction.

Fig. 4. Effective radius $R$ calculated by using Eq.1. The data points are the same as the ones presented in Fig. 3. Due to the fact that the measurements were performed by stepping the magnetic field intensity with a finite step, the effective radius is only measured with a precision of approximately 40nm. The filled (open) dots corresponds to single flux jumps $n=1$ (double flux jumps $n=2$ ). The horizontal lines corresponds to the outer and inner radius determined from the SEM pictures. The arrows indicate sweep direction. It is seen that as the magnetic field intensity is changed the effective radius changes between inner and outer radius of the loop, a changed which depends on sweep direction and magnetic field intensity. The large spread of the data at high magnetic fields corresponds to regions where the amplitude of the oscillations measured by the Hall probe are small. 
Fig. 5. The magnetic field intensity difference $\Delta\left(\mu_{0} H\right)$ between two successive jumps in magnetisation. Given in units of 1.412 Gauss corresponding to a single flux quantum $\Phi_{0}=h / 2 e$ for a loop with a width of $w=630 \mathrm{~nm}$. The plotted jumps are given as a function of magnetic field intensity. The measurement was performed at $T=0.38 \mathrm{~K}$. The positive (negative) flux values corresponds to the case where $\mu_{0} H$ was decreased (increased) during the measurements. Arrows indicate sweep direction. 


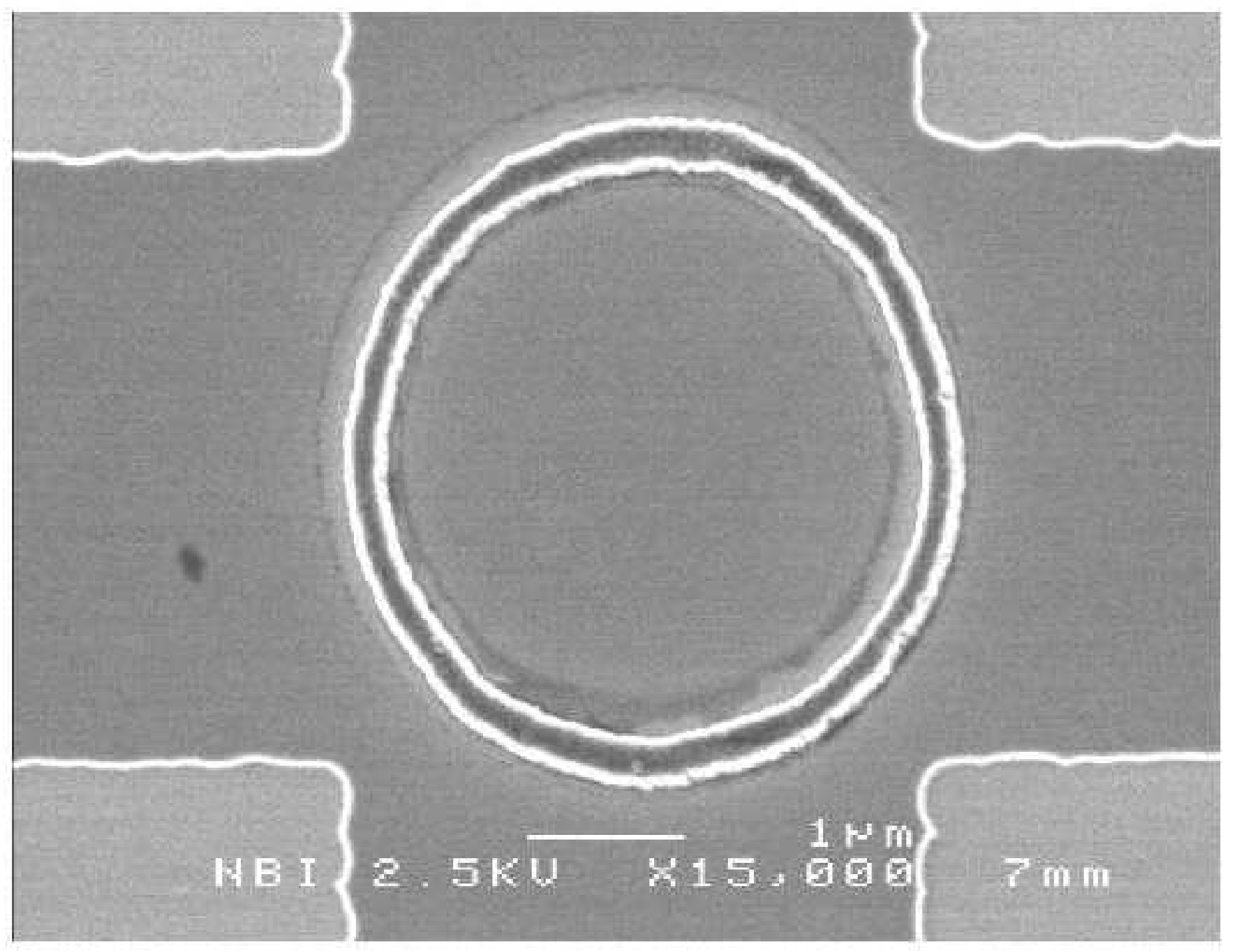




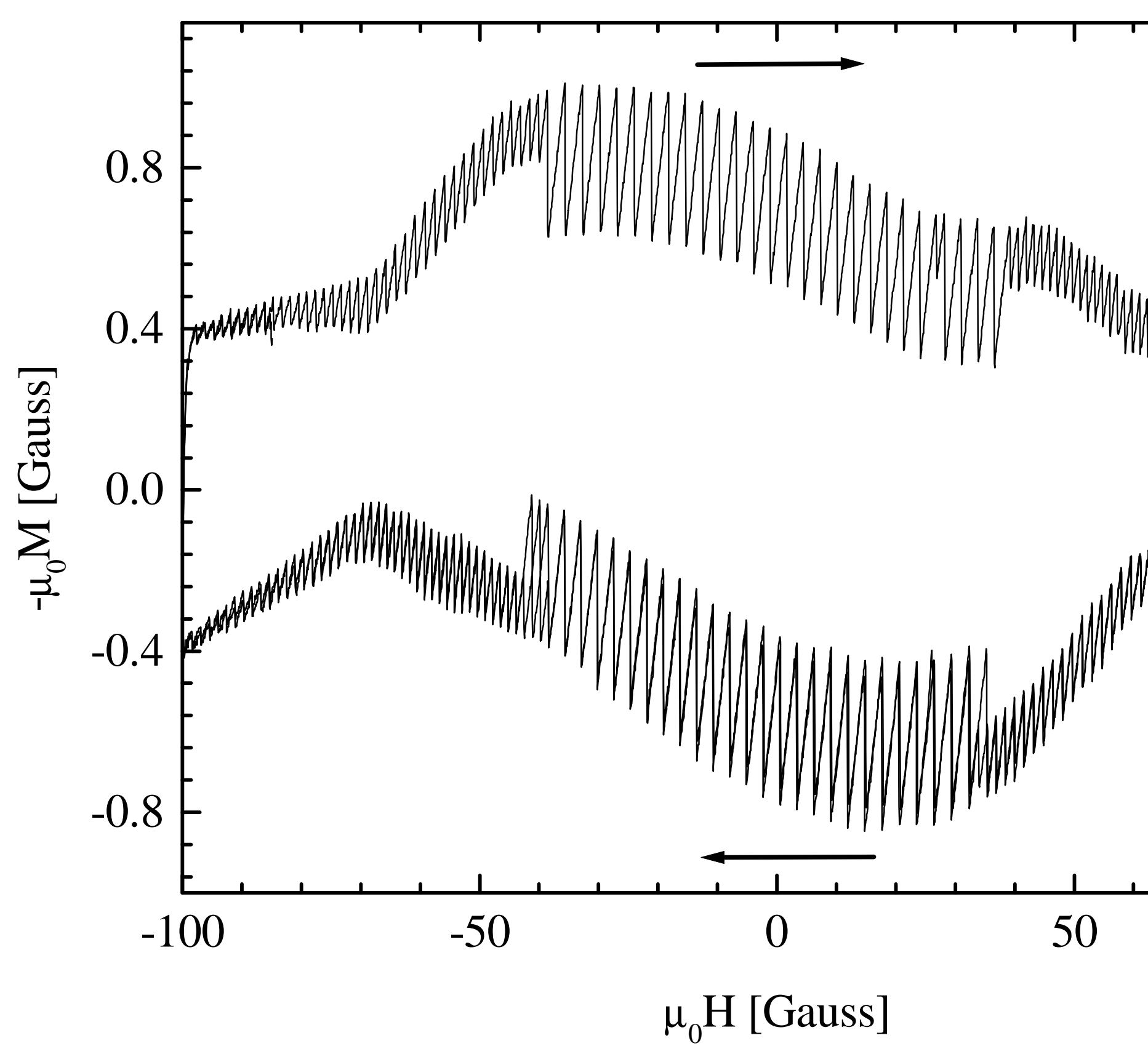




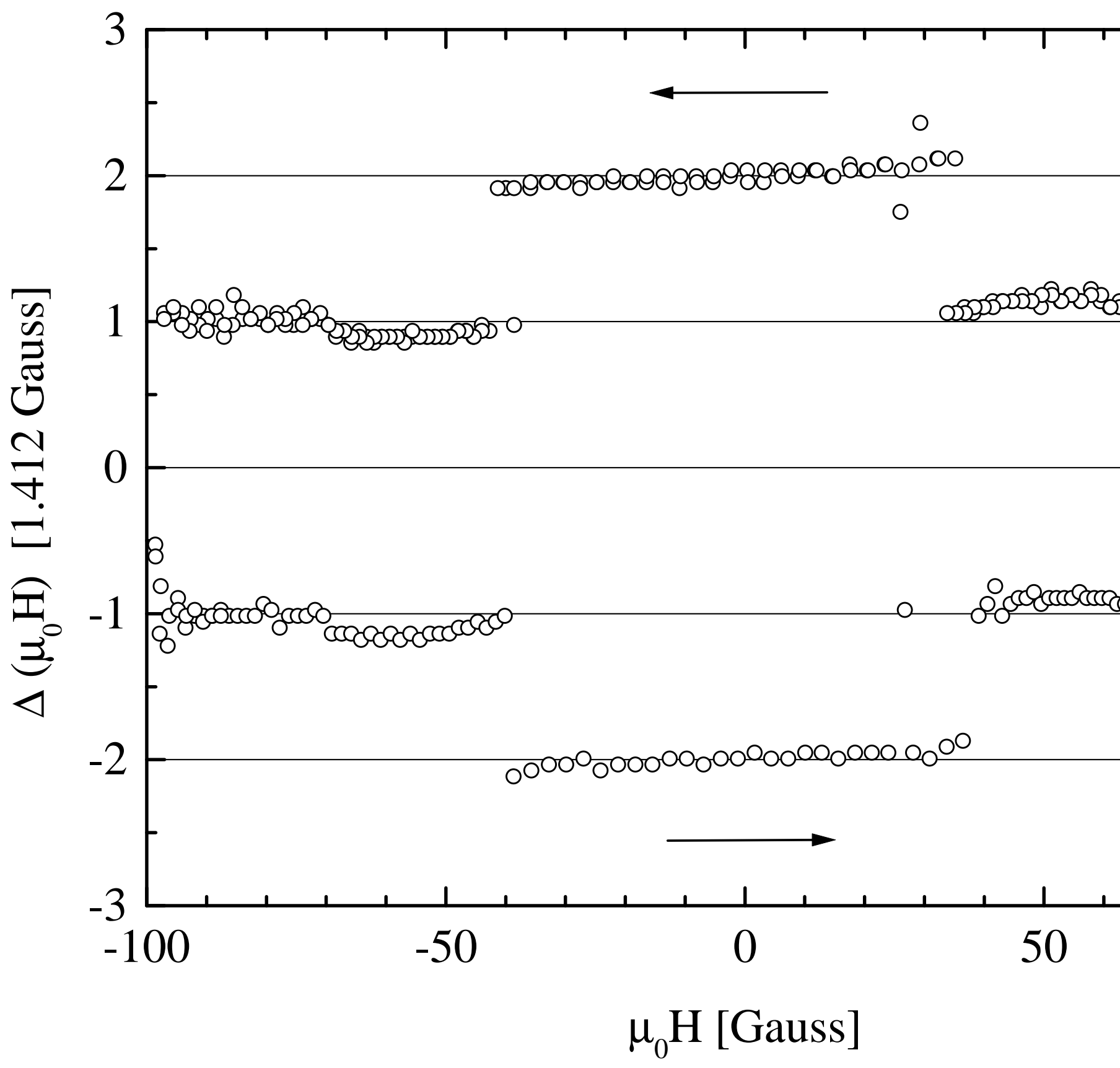




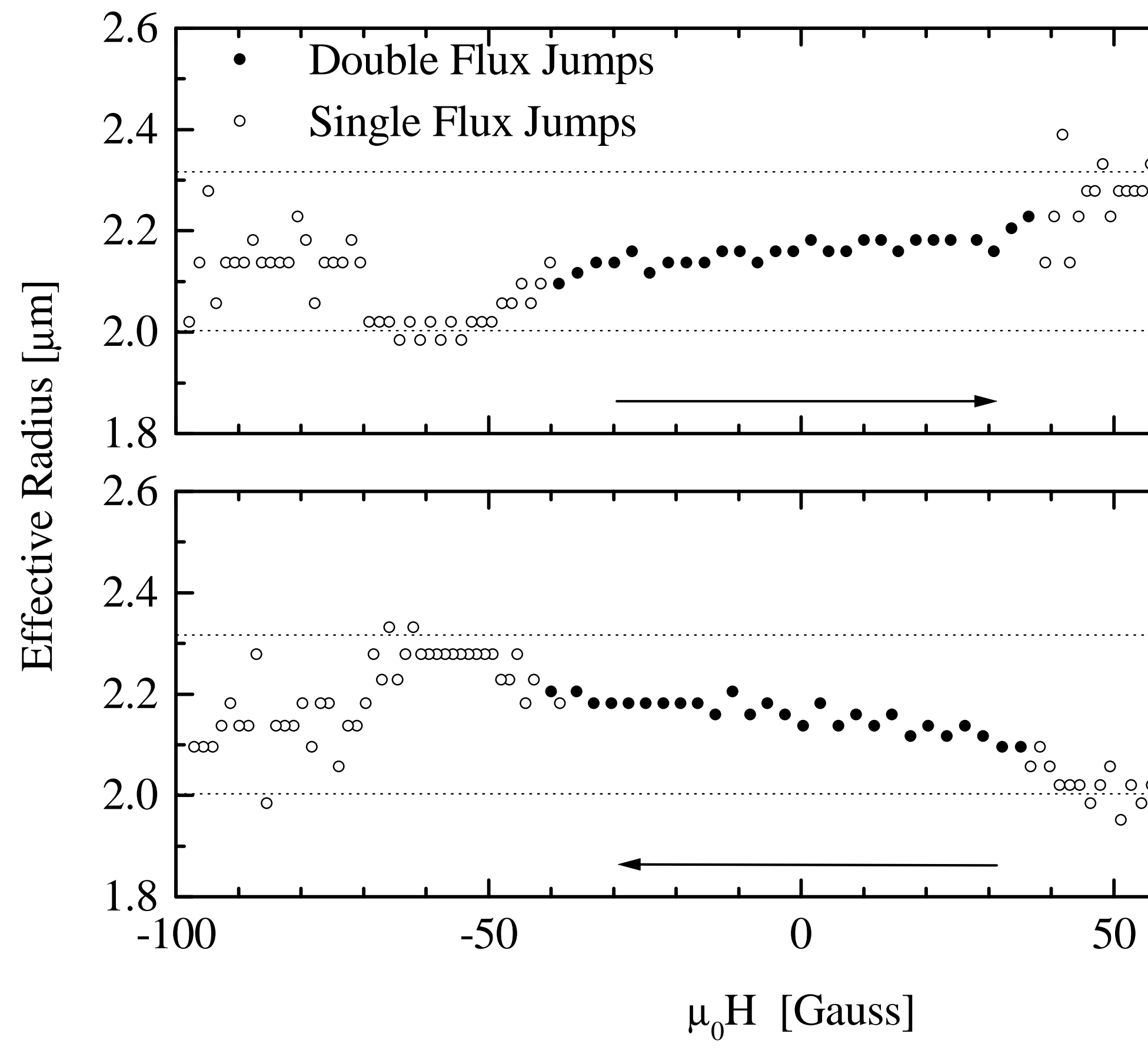




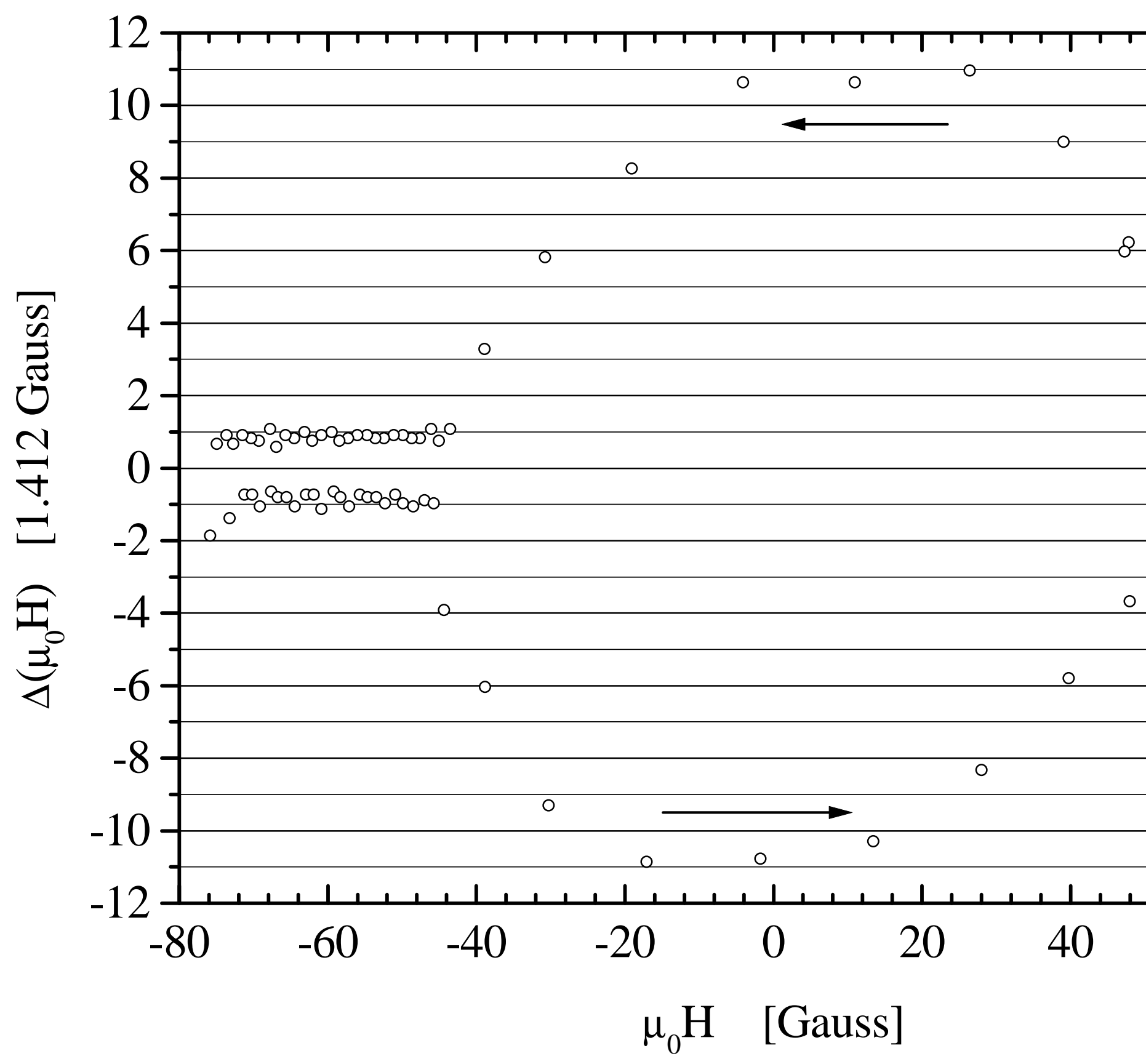

